\title{
Role of vascular mapping in pancreatic resection preoperative planning
}

\begin{abstract}
Celiac axis stenosis may lead to severe liver ischaemia and mortality after pancreatic cephalic resection. $5 \%$ of patients submitted to pancreaticoduodenectomy may have celiac axis stenosis. Atherosclerosis and arcuate ligament syndrome are the most frequent aetiologies. When preoperative radiologic assessment and intraoperative hepatic blood flow assessment suggest celiac axis stenosis, surgical revascularization or, nowadays, endovascular approach is needed before pancreatic head resection.

We present the case of a middle-aged male with an ERCP and cytology diagnosis of peri-ampullary tumor. Review of CT images by the pancreatic surgeons and radiologists in multidisciplinary meeting raises the suspicious of celiac axis stenosis, not previously described. Endovascular approach was attempted although it showed to be technically unfeasible. An aorto-hepatic bypass graft was performed followed by a pancreaticoduodenectomy. Presently, two months after surgery, the patient is under adjuvant chemotherapy. Systematic preoperative assessment by dedicated experts in a multidisciplinary setting leads to a patient specific surgical algorithm strategy which will guide the surgical approach according to the intraoperative findings, minimizing the risk of mortality and morbidity associated to complex surgical procedures.
\end{abstract}

Keywords: pancreatic cancer, pancreatoduodenectomy, celiac axis, aorto-hepatic bypass
Volume 10 Issue 3 - 2019

\author{
António Gomes,' Marta Fragoso,' Marta \\ Baptista, ${ }^{2}$ Gabriela Gasparinho, ${ }^{3}$ Vitor Nunes ${ }^{4}$ \\ 'Department of Surgery, Hospital Prof. Doutor Fernando \\ Fonseca, Portugal \\ ${ }^{2}$ Department of Radiology, Hospital Prof. Doutor Fernando \\ Fonseca, Portugal \\ ${ }^{3}$ Department of Surgical Pathology, Hospital Prof. Doutor \\ Fernando Fonseca, Portugal \\ ${ }^{4}$ Director of Surgery Department, H Hospital Prof. Doutor \\ Fernando Fonseca, Portugal
}

Correspondence: Antonio Pedro Gomes, Hospital Prof. Dr. Fernando Fonseca, IC 19 Venteira, 2720 Amadora, Portugal, Tel +351963750234 ,

Email antonio.p.gomes@hff.min-saude.pt,

apspgomes@gmail.com

Received: September 17,2018 | Published: June 12, 2019
Abbreviations: AHBG, aorto-hepatic bypass graft; AJJC, american joint committee on cancer; $\mathrm{CA}$, celiac axis; $\mathrm{CBD}$, common bile duct; CT scan, computed tomography; ER, emergency Department; ERCP, endoscopic retrograde cholangiopancreatography; GDA, gastroduodenal artery; ICU, intensive care unit; MRI, magnetic resonance imaging; PDA, pancreaticoduodenal(s) artery(ies); SMA, superior mesenteric artery

\section{Introduction}

The celio-mesenteric arterial blood flow assessment is a paradigm in surgical planning and management of cephalic pancreatic cancer. Numerous anatomic variants of the celiac axis (CA) are described, with an estimated prevalence of 10 to $50 \%$. Their awareness is of great importance. ${ }^{1}$ In addition, CA stenosis prevalence is reported in as high as $25 \%$ of all patients and $5 \%$ of those submitted to pancreatoduodenectomy. ${ }^{2-5}$ The main etiologic factors are severe atherosclerosis and arcuate ligament syndrome, ${ }^{2}$ but literature is conflicting regarding their prevalence. Extrinsic tumour compression, dissection, aneurism are less prevalent aetiologies.

In patients with CA blood flow compromise, liver blood flow depends on superior mesenteric artery (SMA) and collateral blood flow through the gastroduodenal artery (GDA) and the inferior pancreatoduodenal arteries (PDA). If GDA and collateral blood flow from inferior PDA are ligated in pancreatic head resection, severe liver ischemia may develop. ${ }^{2,3}$

Several surgical revascularization strategies are described in the literature: splenic to SMA transposition; celiac axis transposition; posterior PDA bypass and aorto-hepatic bypass, with the last one being the most commonly performed. ${ }^{6-8}$ Recently, endovascular therapy is considered the standard of care for CA and SMA disease. However, when associated to pancreatic cancer or an endovascular approach is tried before the pancreatic resection, or a classic open bypass is performed synchronous with the pancreatic resection. Before GDA ligation, we should always assess the intraoperative liver and visceral blood flow by temporary GDA occlusion and pancreatic neck compression. ${ }^{7}$

Preoperative assessment of the arterial system, by CT scan and/or MRI, looking for signs of celiac axis stenosis and anatomical variants is of utmost importance for surgical planning. Although paradigmatic, compromised celiac axis blood flow is somehow underestimated.

\section{Case report}

72-year-old male, admitted to the emergency department (ED) for painless obstructive jaundice (total bilirubin of $2,38 \mathrm{mg} / \mathrm{dL}$ ). Emergent ultrasound revealed dilation of the common bile duct (CBD), with $13 \mathrm{~mm}$, and of the intra-hepatic ducts, distended gallbladder and no visualization of the distal portion of neither the CBD nor the pancreas (Figure 1).

An abdominal and pelvic CT was performed and an unspecific thickening of the distal CBD wall was found (Figure 2). Endoscopic retrograde cholangiopancreatography (ERCP) showed an unspecific inflammation with friability of the Vater papilla and exuberant biliary tree dilation. Drainage of the biliary tree was obtained with a biliary plastic stent. Three weeks later, a second ERCP was performed, with stent removal and multiple ampulla biopsies were collected. Results of Pathology study showed in adenocarcinoma.

In multidisciplinary assessment, our hepato-bilio-pancreatic radiologist highlighted a periampullar resectable tumor and a celiac axis significant stenosis. Diagnostic angiography was then performed, which confirmed the celiac axis stenosis and revascularization was attempted, but proven to be technically infeasible. Surgical resection was decided. Intraoperative hepatic artery flow was severely compromised with GDA occlusion, confirming the celiac axis 
stenosis. A supra-celiac-aorto-hepatic bypass graft (uni-graft K-DV $8 \mathrm{~mm}$ ) followed by a cephalic duodenopancreatectomy with a dunking pancreato-jejunostomy, end-to-side hepatico-jejunostomy and pilorojejunostomy was performed (Figures 3 \& 4).

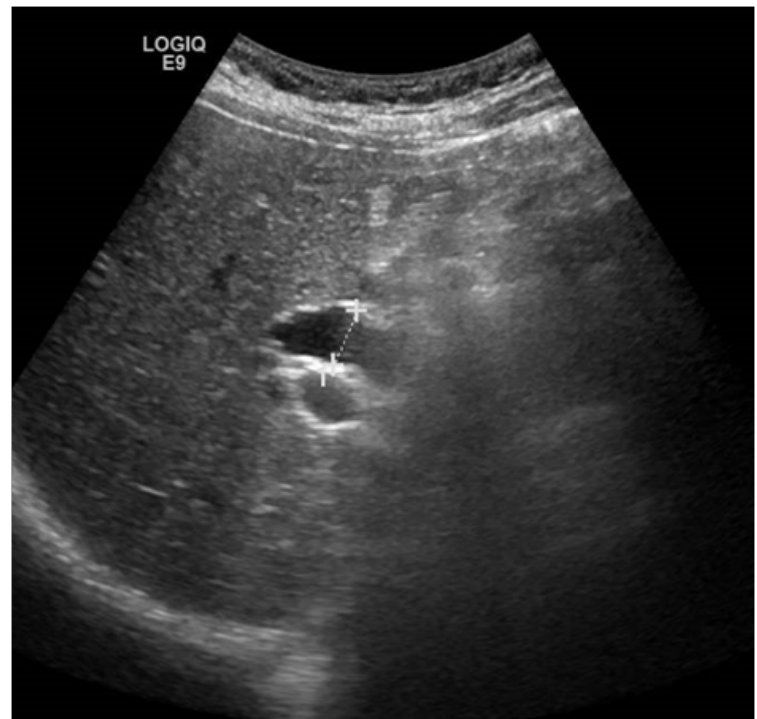

Figure I CBD dilation on ultrasound.
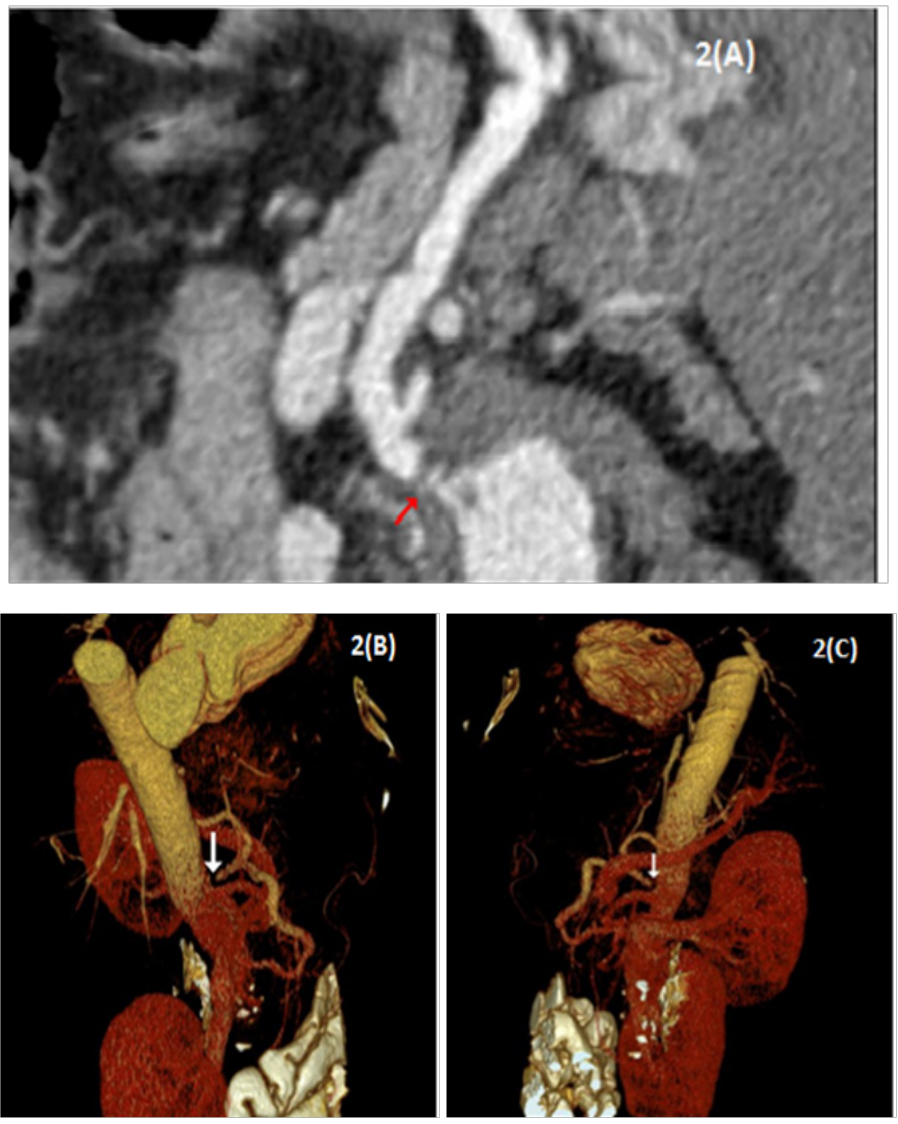

Figure 2 Celiac Axis stenosis in CT-scan.

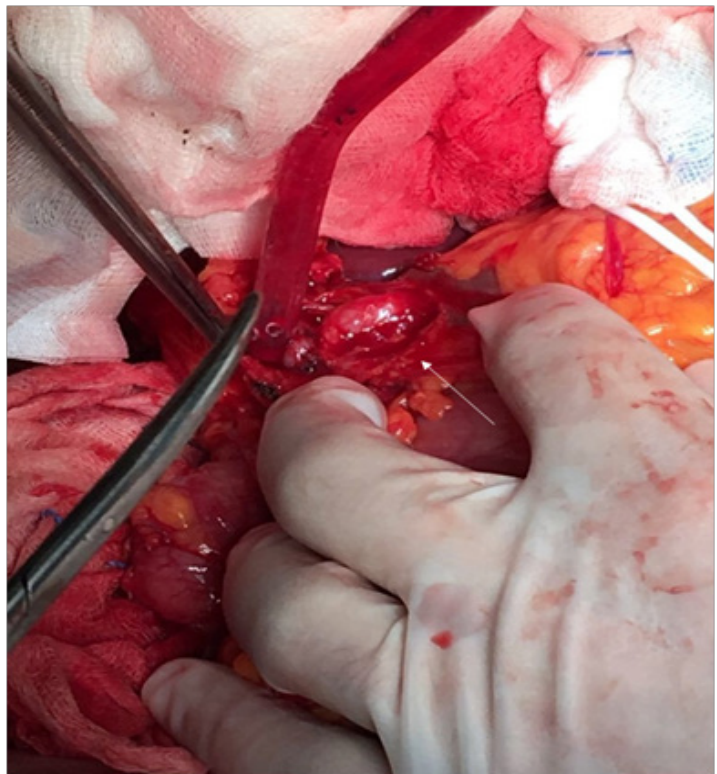

Figure 3 Hepatic artery arteriotomy.

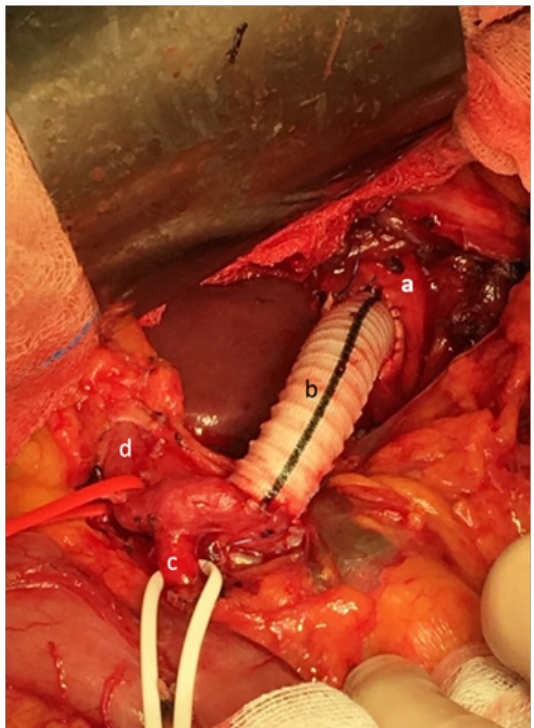

Figure 4 Aortic-hepatic bypass graft.

The postoperative period was complicated by a type a pancreatic fistula. Patient was discharged from the Intensive Care Unit (ICU) on the third postoperative day and from the surgical ward on the eleventh. Surgical pathology analysis showed an adenocarcinoma pT3N1 with perineural and lymphovascular invasion. Surgical resection margins were clear, except for neoplastic tissue found in $<1 \mathrm{~mm}$ of the posterior margin (positive lymph node with extra-capsular involvement) - R1 according to the AJCC $7^{\text {th }}$ Edition (Figures $5 \& 6$ ). ${ }^{9}$

The patient is currently undergoing adjuvant chemotherapy. Twomonth follow-up CT-scan shows no evidence of disease. 


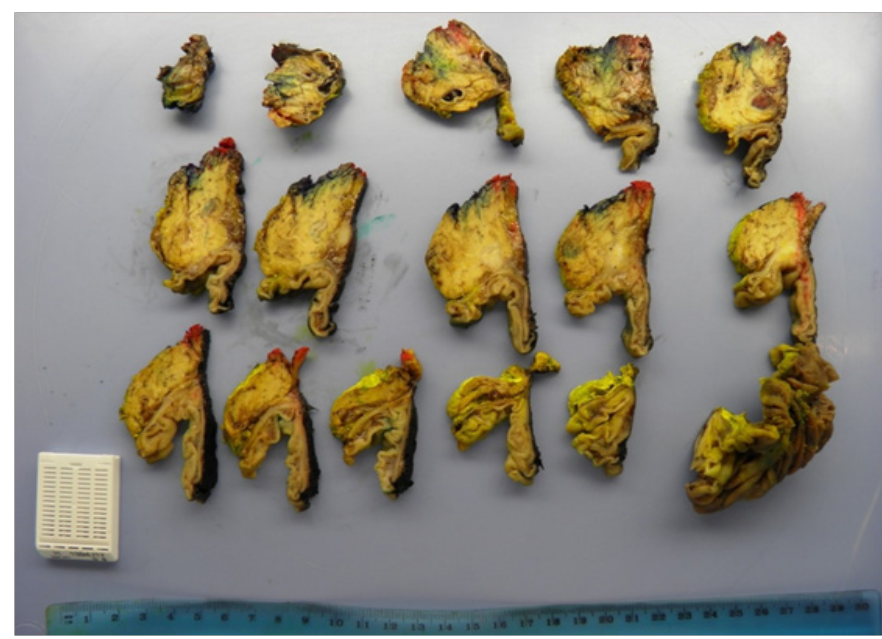

Figure 5 Surgical specimen - macroscopic analysis.

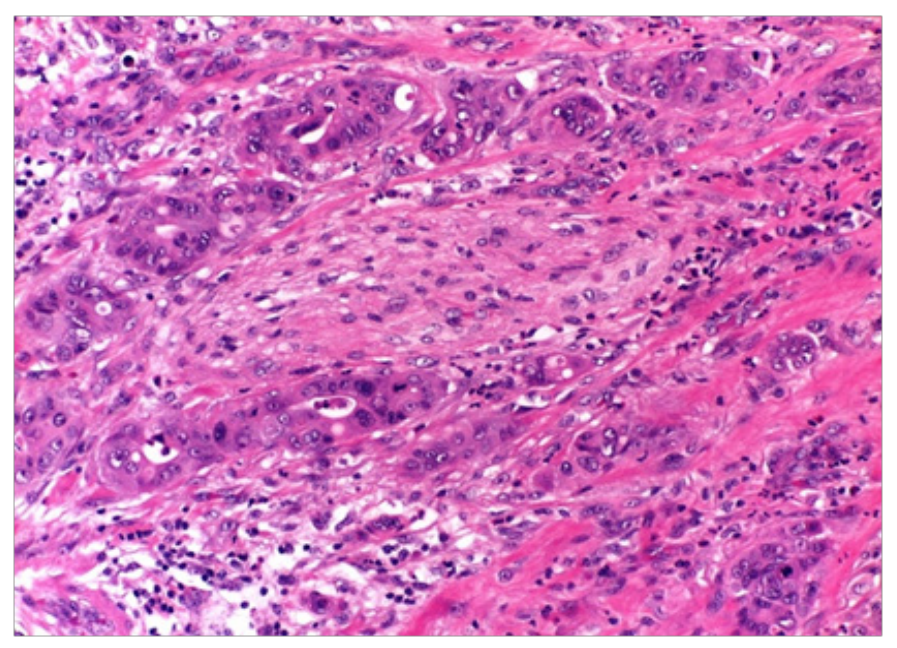

Figure 6 Ampullary Carcinoma.

\section{Discussion}

This is a paradigmatic case with different surgical solutions previously described. However, when specific complex disease became routine practice, some less prevalent issues like anatomic variants and CA stenosis might be underestimated or underappreciated. ${ }^{1}$ Our main aim is to report and emphasize the value of a systematic vascular mapping of celio-mesenteric (and portal-mesenteric) circulation as part of preoperative assessment to establish surgical decision algorithms. These algorithms should fit each patient according to all preoperative data. Angio-CT should systematically be performed and images should be reviewed by the dedicated pancreatic surgeons and radiologists. In this case, signs of CA stenosis were only appreciated after multidisciplinary meeting, and this proved to be one of the key factors to surgical success.

Intraoperative assessment of hepatic artery blood flow after GDA occlusion should be systematically performed. Although not always performed by the authors, other claim that hepatic blood flow should be assessed also after pancreatic neck compression. ${ }^{7}$ Additionally,
Doppler flow measurement which is mandatory in some countries, might have a role may have a role in intraoperative finding of anatomical variants and flow measurement of hepatic artery with transient occlusion of GDA and pancreatic neck..$^{10,11}$

Although histological analysis revealed an R1 (posterior margin) resection, pre-operative staging showed a non-locally advanced resectable disease. This was the reason why multidisciplinary board decided for surgery upfront. Emerging evidence suggests that borderline resectable and even resectable disease might benefit from neoadjuvant systemic chemotherapy. ${ }^{12,13}$

\section{Conclusion}

As a conclusion, systematic preoperative assessment by experts in a multidisciplinary setting leads to a patient specific surgical algorithm strategy. This algorithm previously prepared will guide the surgeons according to the intraoperative findings, minimizing the risk of mortality and morbidity associated to complex surgical procedures. In patients with a performance status fit for complex surgical procedures, vascular reconstructions and bypass enables radical pancreatic resections assuring hepatic, small bowel and gastric perfusion

\section{Acknowledgments}

None.

\section{Conflicts of interest}

There is no financial interest or any conflict of interest.

\section{References}

1. White RD, Weir-McCall JR, Sullivan CM, et al. The celiac axis revisited: anatomic variants, pathologic features, and implications for modern endovascular management. RadioGraphics. 2015;35(3):879-898.

2. Sakorafas GH, Sarr MG, Peros G. Celiac artery stenosis: an underappreciated and unpleasant surprise in patients undergoing pancreaticoduodenectomy. J Am Coll Surg. 2008;206(2):349-356.

3. Farma JM, Hoffman JP. Nonneoplastic celiac axis occlusion in patients undergoing pancreaticoduodenectomy. Am J Surg. 2007;193(3):341-344.

4. Turner KM, Majekodunmi K, Manejwala A, et al. Image findings in celiac artery stenosis due to median arcuate ligament compression: a crucial diagnosis when planning for pancreaticoduodenectomy. $J$ Gastrointest Surg. 2014;18(3):638-640.

5. Hughes T, Chatzizacharias NA, Richards J, et al. Aorto-hepatic bypass graft for repair of an inferior pancreatico-duodenal artery aneurysm associated with coeliac axis occlusion: A case report. Int J Surg Case Rep. 2016;28:131-134.

6. Berselli M, Sperti C, Ballotta E, et al. Pancreaticoduodenectomy with unusual artery reconstruction in a patient with celiac axis occlusion: report of a case. Updates Surg. 2010;62(2):117-120.

7. Machado MC, Penteado S, Montagnini AL, et al. An alternative technique in the treatment of celiac axis stenosis diagnosed during pancreaticoduodenectomy. HPB Surg. 1998;10(6):371-373.

8. Portolani N, Tiberio GA, Coniglio A, et al. Emergency celiac revascularization for supramesocolic ischemia during pancreaticoduodenectomy: report of a case. Surg Today. 2004;34(7):616618.

9. Edge SB BD, Compton CG, Fritz AG, et al. AJCC Cancer Staging Manual. $7^{\text {th }}$ edn. New York: Springer; 2010. 
10. Hirai I, Kimura W, Kamiga M, et al. The significance of intraoperative Doppler ultrasonography in evaluating hepatic arterial flow when assessing the indications for the Appleby procedure for pancreatic body cancer. J Hepatobiliary Pancreat Surg. 2005;12(1):55-60.

11. Tori M, Nakahara M, Akamatsu H, et al. Significance of intraoperative monitoring of arterial blood flow velocity and hepatic venous oxygen saturation for performing minimally invasive surgery in a patient with multiple calcified pancreaticoduodenal aneurysms with celiac artery occlusion. J Hepatobiliary Pancreat Surg. 2006;13(5):472-476.
12. Ettrich TJ, Berger AW, Perkhofer L, et al. Neoadjuvant plus adjuvant or only adjuvant nab-paclitaxel plus gemcitabine for resectable pancreatic cancer - the NEONAX trial (AIO-PAK-0313), a prospective, randomized, controlled, phase II study of the AIO pancreatic cancer group. BMC Cancer. 2018;18(1):1298.

13. Klaiber U, Leonhardt CS, Strobel O, et al. Neoadjuvant and adjuvant chemotherapy in pancreatic cancer. Langenbecks Arch Surg. 2018;403(8):917-932. 